Pacific Journal of Mathematics

NOTE ON THE PWB-METHOD IN THE NONLINEAR GAS

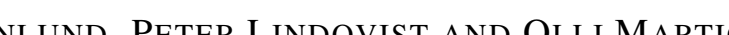




\title{
NOTE ON THE PWB-METHOD IN THE NON-LINEAR CASE
}

\author{
S. GRANLund, P. Lindqvist ANd O. Martio
}

The Perron-Wiener-Brelot (PWB) method is applied to an important nonlinear situation. Unbounded subsolutions, their approximation and a counterpart of the harmonic measure are considered.

Introduction. The Perron-Wiener-Brelot (PWB-) method as introduced by $\mathbf{O}$. Perron $[\mathbf{P}]$ and refined by several mathematicians is wellknown in Potential Theory and it is mainly used in the theory of harmonic functions although it has a wider scope of applications [CC]. The PWBmethod was generalized by E. Beckenbach and L. Jackson [BJ, J] to the non-linear situation. Their approach used the strong maximum principle for the difference of two solutions [BJ, Postulate 2]. The purpose of this note is to show that the PWB-method can be employed without this assumption in certain important non-linear cases. We are also able to deal with unbounded subsolutions.

We consider weak solutions, called $F$-extremals, of an Euler equation

$$
\nabla \cdot \nabla_{h} F(x, \nabla u)=0,
$$

where the variational kernel $F: G \times R^{n} \rightarrow R$ satisfies the assumptions:

(a) For each $\varepsilon>0$ there is a closed set $C$ in the domain $G \subset R^{n}$ such that $m(G \backslash C)<\varepsilon$ and $F \mid C \times R^{n}$ is continuous.

(b) For a.e. $x \in G$ the function $h \mapsto F(x, h)$ is strictly convex and differentiable in $R^{n}$.

(c) There are $0<\alpha \leq \beta<\infty$ such that for a.e. $x \in G$

$$
\alpha|h|^{n} \leq F(x, h) \leq \beta|h|^{n},
$$

$h \in R^{n}$.

(d) For a.e. $x \in G$

$$
F(x, \lambda h)=|\lambda|^{n} F(x, h),
$$

$\lambda \in R, h \in R^{n}$.

For a thorough analysis of the above assumptions see [GLM1]. Some of the assumptions are not necessary for the constructions. The exponent $n$ in (c) is essential for applications in conformal geometry, cf. [GLM1-2]. 
A function $u \in C(G) \cap \operatorname{loc} W_{n}^{1}(G)$, i.e. $u$ is $A C L^{n}$, is called an $F$-extremal in $G$ if for all domains $D \subset \subset G$

where

$$
I_{F}(u, D)=\inf _{v \in \mathscr{F}_{u}} I_{F}(v, D)
$$

$$
I_{F}(v, D)=\int_{D} F(x, \nabla v(x)) d m(x)
$$

is the variational integral with the kernel $F$ and

$$
\mathscr{F}_{u}=\left\{v \in C(\bar{D}) \cap W_{n}^{1}(D) \mid v=u \text { in } \partial D\right\} .
$$

A function $u$ is an $F$-extremal if and only if $u \in C(G) \cap \operatorname{loc} W_{n}^{1}(G)$ is a solution of (1.1) in the weak sense.

An upper semi-continuous function $u: G \rightarrow R \cup\{-\infty\}$ is called a sub- $F$-extremal if $u$ satisfies the $F$-comparison principle in $G$, i.e. if $D \subset \subset G$ is a domain and $h \in C(\bar{D})$ is an $F$-extremal in $D$, then $h \geq u$ in $\partial D$ implies $h \geq u$ in $D$. A function $u: G \rightarrow R \cup\{\infty\}$ is a super-F-extremal if $-u$ is a sub- $F$-extremal. For the basic properties of $F$-extremals and sub- $F$-extremals we refer to [GLM1].

Let $G \subset R^{n}$ be a domain and let $f: \partial G \rightarrow R \cup\{ \pm \infty\}$ be any function. The fundamental concepts in the PWB-method are the upper and lower classes $\mathscr{U}_{f}$ and $\mathscr{L}_{f}$ determined by $f$. Our first theorem, Theorem 2.2, states in the complete analogy with the PWB-method that the function

$$
\underline{H}_{f}(x)=\sup \left\{u(x) \mid u \in \mathscr{L}_{f}\right\}
$$

is either $+\infty,-\infty$ or an $F$-extremal in $G$. The proof differs in several aspects from the classical proof, cf. e.g. [H]. First, a method like the Poisson modification of a sub-F-extremal is needed and since no Poisson formula is available in the non-linear case, our modification is based on approximation and on the solvability of the Dirichlet's problem in balls. The crucial step in the proof is to show that the function $\underline{H}_{f}$ is continuous if it is not $+\infty$ or $-\infty$. The proof is based on a uniform Hölder-estimate, see [GLM1, Theorem 4.7], which is quite similar to Harnack's inequality. Moreover, the proof for Theorem 2.2 uses a uniform approximation argument, Lemma 2.14, for the function $\underline{H}_{f}$ and Harnack's principle several times.

The rest of the paper is devoted to applications of the PWB-method and to byproducts of the method. In Chapter 3 we develop the barrier method for the non-linear case. Here the method works as in the linear case. Moreover, this method gives a necessary and sufficient condition for the solvability of the Dirichlet problem with continuous boundary values. In the non-linear case the best condition for solvability has been the 
celebrated Wiener criterion, see [Maz], [GZ]. In the harmonic case these conditions are equivalent but this is not known in the non-linear situation.

Approximation of sub- $F$-extremals by means of regular sub- $F$-extremals is studied in Chapter 4 . These results which are usually proved using a simple convolution argument, cf. [R], are more difficult to obtain in the non-linear case and we need a solution to an obstacle problem in the calculus of variations, cf. [GLM1, Theorem 5.15]. As a consequence we especially show that a bounded subharmonic function in a plane domain belongs to the Sobolev space loc $W_{2}^{1}$. These results are needed in the variational interpretation of subharmonicity and, more generally, sub- $F$ extremality.

Chapter 5 is devoted to the construction of the $F$-harmonic measure in general domains. This concept has turned out useful in studying the boundary behavior of $F$-extremals, see [GLM2], [GLM3].

Our notation is standard and generally as in [GLM1].

2. The Perron-Wiener-Brelot method. Suppose that $G \subset R^{n}$ is a domain and that $f: \partial G \rightarrow R \cup\{ \pm \infty\}$ is a function.

2.1. Definition. The lower class $\mathscr{L}_{f}$ consists of the functions $u$ : $G \rightarrow R \cup\{-\infty\}$ for which

(a) $u$ is a sub- $F$-extremal in $G$,

(b) $u$ is bounded above,

(c) $\varlimsup_{x \rightarrow y} u(x) \leq f(y)$ for all $y \in \partial G$,

(d) there is a compact set $K_{u} \subset R^{n}$ such that $u \leq 0$ in $G \backslash K_{u}$. The upper class $\mathscr{U}_{f}$ is defined analogously via super- $F$-extremals.

Let $\bar{H}_{f}=\inf \left\{u \mid u \in \mathscr{U}_{f}\right\}$ and $\underline{H}_{f}=\sup \left\{u \mid u \in \mathscr{L}_{f}\right\}$. The next theorem is fundamental for the PWB-method.

2.2. THEOREM. The function $\underline{H}_{f}$ satisfies one of the following conditions:

(i) $\underline{H}_{f}$ is an F-extremal in $G$,

(ii) $\underline{H}_{f}=\infty$ in $G$,

(iii) $\underline{H}_{f}=-\infty$ in $G$.

The same is true for the funciton $\bar{H}_{f}$.

Some auxiliary results are needed in the proof of the above theorem. The so-called $F$-comparison principle is a basic tool.

2.3. LEMMA. Let $G \subset R^{n}$ be a bounded open set, $u$ a sub-F-extremal and $v$ a super-F-extremal in $G$. Suppose

$$
\varlimsup_{x \rightarrow y} u(x) \leq \lim _{x \rightarrow y} v(x)
$$


for all $y \in \partial G$. If the left and right-hand sides are neither $\infty$ nor $-\infty$ at the same time, then $u \leq v$ in $G$.

Proof. Fix any $x \in G$. We will show that $u(x) \leq v(x)$. Let $\varepsilon>0$ and consider the open set $H=\{y \in G \mid u(y)<v(y)+\varepsilon\}$. There exists a regular domain $D_{\varepsilon}, \bar{D}_{\varepsilon} \subset G$, such that $x \in D_{\varepsilon}$ and $\partial D_{\varepsilon} \subset H$. Choose a decreasing sequence $\varphi_{i} \in C^{\infty}(G)$ and an increasing sequence $\psi_{i} \in C^{\infty}(G)$ such that $\varphi_{i} \rightarrow u$ and $\psi_{i} \rightarrow v+\varepsilon$. Since $\partial D_{\varepsilon}$ is compact we have $\varphi_{i}<\psi_{i}$ on $\partial D_{\varepsilon}$ for some $i \in N$. Let $h_{i}^{1}$ and $h_{i}^{2}$ be $F$-extremals such that $h_{i}^{1} \mid \partial D_{\varepsilon}=$ $\varphi_{i}\left|\partial D, h_{i}^{2}\right| \partial D_{\varepsilon}=\psi_{i} \mid \partial D$. It follows from [GLM1, Definition 5.1] that

$$
u \leq h_{i}^{1} \leq h_{i}^{2} \leq v+\varepsilon \text { in } D_{\varepsilon} .
$$

Since $x \in D_{\varepsilon}$ and $\varepsilon>0$ was arbitrary, we obtain the desired inequality $u(x) \leq v(x)$.

The $F$-comparison principle yields:

\subsection{LEMMA. $\underline{H}_{f} \leq \bar{H}_{f}$.}

The Poisson modification of a subharmonic function so as to be harmonic over part of its domain is a basic operation in the classical potential theory. In the proof of Theorem 2.2 we employ a similar modification method for sub- $F$-extremals, cf. [R].

2.6. Modification of sub-F-extremals. Suppose that $G \subset R^{n}$ is a domain and that $u: G \rightarrow R \cup\{-\infty\}$ is a sub-F-extremal. Let $\bar{B} \subset G$ be a ball. We modify the sub- $F$-extremal by an approximation argument. Since $u$ is upper semicontinuous in $G$, there exists a sequence $\varphi_{i} \in C^{\infty}(G)$ such that $\varphi_{1} \geq \varphi_{2} \geq \cdots \geq u$ in $\bar{B}$ and $\lim _{i \rightarrow \infty} \varphi_{i}=u$ in $\bar{B}$.

Choose $F$-extremals $h_{i}$ in $\bar{B}$ such that $h_{i}\left|\partial B=\varphi_{i}\right| \partial B$ and $h_{i} \in C(\bar{B})$ $\cap W_{n}^{1}(B)$. By Lemma 2.3, $h_{1} \geq h_{2} \geq \cdots \geq u$ in $\bar{B}$. The function $h=$ $\lim _{i \rightarrow \infty} h_{i}$ is an $F$-extremal or identically $-\infty$ in $B$, see [GLM1, Theorem 4.22]. For any $\zeta \in \partial B$

$$
\varlimsup_{\substack{x \rightarrow \zeta \\ x \in B}} h(x) \leq \varlimsup_{\substack{x \rightarrow \zeta \\ x \in B}} h_{i}(x)=\varphi_{i}(\zeta), \quad i=1,2,3, \ldots,
$$

and thus

Write

$$
\varlimsup_{\substack{x \rightarrow \zeta \\ x \in B}} h(x) \leq u(\zeta)
$$

$$
P(u, B)(x)= \begin{cases}u(x), & x \in G \backslash B \\ h(x), & x \in B\end{cases}
$$


It is easy to see that $P(u, B)$ is independent of the sequence $\varphi_{i}$, although this fact is not needed in the sequel.

Now $P(u, B) \geq u$ in $G$ and we shall prove that the function $P(u, B)$ is a sub- $F$-extremal. For that purpose an auxiliary result is needed.

2.8. LEMMA. A sub-F-extremal $u$ is identically $-\infty$ if and only if it is $-\infty$ in some nonempty open subset of $G$.

Proof. Write $H=\inf \{x \in G \mid u(x)=-\infty\}$ and suppose $H \neq G$. Let $x_{0} \in \partial H \cap G$ and choose $\delta>0$ such that $\bar{B}^{n}\left(x_{0}, \delta\right) \subset G$ and $S^{n-1}\left(x_{0}, \delta\right) \cap H \neq \varnothing$. Pick a closed cap $K \subset S^{n-1}\left(x_{0}, \delta\right) \cap H$ and denote the $F$-harmonic measure $\omega\left(K, B^{n}\left(x_{0}, \delta\right) ; F\right)$ by $h$. It follows from [GLM2, Theorem 4.10] that $h$ is not identically zero. Let $\alpha>0$ and consider the $F$-extremal $v=M-\alpha h$ where $M=\sup _{B^{n}\left(x_{0}, \delta\right)} u$. Now

$$
\lim _{x \rightarrow y} v(x) \geq \varlimsup_{x \rightarrow y} u(x)
$$

for all $y \in S^{n-1}\left(x_{0}, \delta\right)$ and the left-hand side is finite. Hence by Lemma 2.3, $v \geq u$ in $B^{n}\left(x_{0}, \delta\right)$. Letting $\alpha \rightarrow \infty$ we obtain $u(x)=-\infty$ for all $x \in B^{n}\left(x_{0}, \delta\right)$, a contradiction since $B^{n}\left(x_{0}, \delta\right)$ contains points not in $H$. The lemma follows.

We are ready to prove

2.9. LeMMA. The function $\mathscr{U}=P(u, B)$ is a sub-F-extremal in $G$.

Proof. If $h$ is identically $-\infty$, so is $u$ by Lemma 2.8 and there is nothing to prove. Otherwise, $h$ is an $F$-extremal and we first show that $\mathscr{U}$ is upper semicontinuous. We need only consider points $\zeta \in \partial B$. Now

$$
\varlimsup_{\substack{x \rightarrow \zeta \\ x \in G \backslash B}} \mathscr{U}(x)=\varlimsup_{\substack{x \rightarrow \zeta \\ x \in G \backslash B}} u(x) \leq u(\zeta)=\mathscr{U}(\zeta)
$$

By combining (2.7) and the inequality above it readily follows that $\mathscr{U}$ is upper semicontinuous.

Next we prove that $\mathscr{U}$ satisfies the $F$-comparison principle in $G$. Suppose that $D \subset \subset G$ is a domain and that $H \in C(\bar{D})$ is an $F$-extremal in $D$ with $H|\partial D \geq \mathscr{U}| \partial D$. We will show that $H \geq \mathscr{U}$ in $D$. Now $H \geq u$ in $D$, since $\mathscr{U} \geq u$ in $G$ and $u$ is a sub- $F$-extremal. Let $\zeta \in \partial(D \cap B)$. Now

$$
H(\zeta) \geq u(\zeta) \geq \varlimsup_{\substack{x \rightarrow \zeta \\ x \in D \cap B}} h(x)
$$


and hence

$$
\lim _{\substack{x \rightarrow \zeta \\ x \in D \cap B}} H(x) \geq \varlimsup_{\substack{x \rightarrow \zeta \\ x \in D \cap B}} h(x) .
$$

Lemma 2.3 implies $H \geq h=\mathscr{U}$ in $D \cap B$. Consequently $H \geq \mathscr{U}$ in the whole $D$ as desired.

2.10 Three lemmas for $\underline{H}_{f}$. In what follows we shall only consider the function $\underline{H}_{f}$. The lemmas and proofs for $\bar{H}_{f}$ are similar. First we prove that it is possible to replace $\mathscr{L}_{f}$ by a subfamily, which is bounded from below in compact subsets of $G$. This new family gives the same $\underline{H}_{f}$.

2.11. LemMA. Suppose $K \subset G$ is compact and $\underline{H}_{f}$ is not identically $-\infty$. Then there exists $\mathscr{L}_{K} \subset \mathscr{L}_{f}$ such that $\mathscr{L}_{K}$ is bounded from below in $K$ and $\underline{H}_{f}=\sup \mathscr{L}_{K}$.

Proof. There exists $u_{0} \in \mathscr{L}_{f}$ such that $u_{0}$ is not identically $-\infty$. Choose a finite cover $\left\{B^{n}\left(x_{1}, R_{x_{1}}\right), \ldots, B^{n}\left(x_{k}, R_{x_{k}}\right)\right\}$ of $K$ such that $\bar{B}^{n}\left(x_{i}, 2 R_{x_{i}}\right) \subset G$ for $i=1, \ldots, k$, and let $\mathscr{U}_{x_{i}}=P\left(u_{0}, B^{n}\left(x_{i}, 2 R_{x_{i}}\right)\right), i=$ $1, \ldots, k$. Lemma 2.9 yields $\mathscr{U}_{x_{i}} \in \mathscr{L}_{f}$ and Lemma 2.8 shows that $\mathscr{U}_{x_{i}}>-\infty$ in $B^{n}\left(x_{i}, 2 R_{x_{i}}\right), i=1, \ldots, k$. Since $\mathscr{U}_{x_{i}}$ is an $F$-extremal in $B^{n}\left(x_{i}, 2 R_{x_{i}}\right)$ the continuity of $\mathscr{U}_{x_{i}}$ gives $M_{x_{i}}<\infty$ such that $\mathscr{U}_{x_{i}}>-M_{x_{i}}$ in $B^{n}\left(x_{i}, R_{x_{i}}\right)$. Choose $\mathscr{L}_{K}=\left\{\max \left\{u, \mathscr{U}_{x_{1}}, \ldots, \mathscr{U}_{x_{k}}\right\} \mid u \in \mathscr{L}_{f}\right\}$. Observe that for all $u \in \mathscr{L}_{K}$ we have $u \geq \min \left\{-M_{x_{1}}, \ldots,-M_{x_{k}}\right\}$ in $K$. Let $u \in \mathscr{L}_{f}$. Then there exists $u^{*} \in \mathscr{L}_{K}$ such that $u^{*} \geq u$ in $G$. It follows that $\sup \mathscr{L}_{K}=\underline{H}_{f}$.

The next lemma is the basic step for the proof of Theorem 2.2 .

2.12. LEMMA. If $\underline{H}_{f}$ is locally bounded above, then $\underline{H}_{f}$ is continuous or identically $-\infty$.

Proof. Let $\varepsilon>0$ and assume that $\underline{H}_{f}$ is not identically $-\infty$. We will show that there is $r>0$ such that

$$
\left|\underline{H}_{f}\left(x_{1}\right)-\underline{H}_{f}\left(x_{2}\right)\right|<\varepsilon \text { for } x_{1}, x_{2} \in B^{n}\left(x_{0}, r\right) .
$$

Fix a ball $\bar{B}^{n}\left(x_{0}, R\right) \subset G$ and let $K=\bar{B}^{n}\left(x_{0}, R\right)$. By Lemma 2.11 we can restrict to the class $\mathscr{L}_{K}$ and there is a constant $0<M_{K}<\infty$ such that $|u(x)|<M_{K}, x \in K$, for all $u \in \mathscr{L}_{K}$. Suppose $x_{1}, x_{2} \in B^{n}\left(x_{0}, r\right) \subset$ $B^{n}\left(x_{0}, R\right)$, where $r>0$ will be fixed later. Assume, for example, that $\underline{H}_{f}\left(x_{2}\right) \geq \underline{H}_{f}\left(x_{1}\right)$. We can choose a sequence of functions $u_{i} \in \mathscr{L}_{K}$ 
such that $\lim _{i \rightarrow \infty} u_{i}\left(x_{2}\right)=\underline{H}_{f}\left(x_{2}\right)$. Consider the functions $\mathscr{U}_{i}=$ $P\left(u_{i}, B^{n}\left(x_{0}, R\right)\right)$. Again we have

$$
\lim _{i \rightarrow \infty} \mathscr{U}_{i}\left(x_{2}\right)=\lim _{i \rightarrow \infty} P\left(u_{i}, B^{n}\left(x_{0}, R\right)\right)\left(x_{2}\right) .
$$

Choose $i_{0} \in N$ so large that $\underline{H}_{f}\left(x_{2}\right)-\mathscr{U}_{i}\left(x_{2}\right) \leq \varepsilon / 2$ for $i>i_{0}$. Now

$$
\begin{aligned}
0 & \leq \underline{H}_{f}\left(x_{2}\right)-\underline{H}_{f}\left(x_{1}\right)<\mathscr{U}_{i}\left(x_{2}\right)+\frac{\varepsilon}{2}-\mathscr{U}_{i}\left(x_{1}\right) \\
& \leq \operatorname{osc}\left(\mathscr{U}_{i}, B^{n}\left(x_{0}, r\right)\right)+\frac{\varepsilon}{2} .
\end{aligned}
$$

It is possible to choose $r$ independent of $i$ such that $\operatorname{osc}\left(\mathscr{U}_{i}, B^{n}\left(x_{0}, r\right)\right)<\varepsilon / 2$. Since $\mathscr{U}_{i}$ is an $F$-extremal in $B^{n}\left(x_{0}, R\right)$, this follows from the Hölder-estimate of $F$-extremals

$$
\operatorname{osc}\left(\mathscr{U}_{i}, B^{n}\left(x_{0}, r\right)\right) \leq c\left(\frac{r}{R}\right)^{\kappa} \operatorname{osc}\left(\mathscr{U}_{i}, B^{n}\left(x_{0}, R\right)\right) \leq 2 c\left(\frac{r}{R}\right)^{\kappa} M_{K},
$$

cf. [GLM1, Theorem 4.7]. In the same way (2.13) can be proved if $\underline{H}_{f}\left(x_{2}\right) \leq \underline{H}_{f}\left(x_{1}\right)$.

2.14. Lemma. Suppose that $C \subset G$ is compact and that $\underline{H}_{f}$ is locally bounded from above in $G$. Then for arbitrary $\varepsilon>0$ there exists $v_{\varepsilon} \in \mathscr{L}_{f}$ such that

$$
\underline{H}_{f}(x) \leq v_{\varepsilon}(x)+\varepsilon \quad \text { for } x \in C .
$$

Proof. By Lemma 2.12 there are two possibilities: either $\underline{H}_{f}$ is identically $-\infty$ or continuous. In the first case choose $v_{\varepsilon} \equiv-\infty$. In the second case for each $x \in C$ choose $\bar{B}^{n}\left(x, 2 R_{x}\right) \subset G$. Let

$$
K=\overline{\bigcup_{x \in C} B^{n}\left(x, R_{x}\right)}
$$

and replace $\mathscr{L}_{f}$ by the class $\mathscr{L}_{K}$. Now $\mathscr{L}_{K}$ is uniformly bounded on the compact subset $K$ of $G$.

For all $x \in C$ there exists $u_{x} \in \mathscr{L}_{K}$ such that $u_{x}(x) \geq \underline{H}_{f}(x)-\varepsilon / 3$. Consider $\mathscr{U}_{x}=P\left(u_{x}, B^{n}\left(x, R_{x}\right)\right)$. There exists $M_{K}>0$ such that $\operatorname{osc}\left(\mathscr{U}_{x}, B^{n}\left(x, R_{x}\right)\right) \leq M_{K}$. Let $x \in K$. Since $\underline{H}_{f}$ is continuous and the Hölder-estimate [GLM1, Theorem 4.7] is valid for $\mathscr{U}_{x}$ in $B^{n}\left(x, R_{x}\right)$ we can choose a ball $B^{n}\left(x, r_{x}\right), 0<r_{x}<R_{x}$, such that

$$
\operatorname{osc}\left(\mathscr{U}_{x}, B^{n}\left(x, r_{x}\right)\right)<\frac{\varepsilon}{3}, \quad \operatorname{osc}\left(\underline{H}_{f}, B^{n}\left(x, r_{x}\right)\right)<\frac{\varepsilon}{3} .
$$

Now $\left\{B^{n}\left(x, r_{x}\right) \mid x \in C\right\}$ is an open cover for $C$ and there is a finite subcover $\left\{B^{n}\left(x_{1}, r_{x_{1}}\right), \ldots, B^{n}\left(x_{k}, r_{x_{k}}\right)\right\}$ of $C$. For $y \in B^{n}\left(x_{i}, r_{x_{i}}\right), i \in$ 
$\{1, \ldots, k\}$, we have

$$
\begin{aligned}
\underline{H}_{f}(y)-\mathscr{U}_{x_{i}}(y)= & \left(\underline{H}_{f}(y)-\underline{H}_{f}\left(x_{i}\right)\right)+\left(\underline{H}_{f}\left(x_{i}\right)-\mathscr{U}_{x_{i}}\left(x_{i}\right)\right) \\
& +\left(\mathscr{U}_{x_{i}}\left(x_{i}\right)-\mathscr{U}_{x_{i}}(y)\right) \\
& <\frac{\varepsilon}{3}+\frac{\varepsilon}{3}+\frac{\varepsilon}{3}=\varepsilon .
\end{aligned}
$$

The function $v_{\varepsilon}=\max \left\{\mathscr{U}_{x_{1}}, \ldots, \mathscr{U}_{x_{k}}\right\}$ is a sub-F-extremal [GLM1, Lemma 5.2] and has the desired property.

Proof of Theorem 2.2. Assume first that $\underline{H}_{f}$ is not locally bounded from above. Then there are a sequence of functions $u_{i} \in \mathscr{L}_{f}$ and a sequence of points $x_{i} \in G$ such that $\lim _{i \rightarrow \infty} u_{i}\left(x_{i}\right)=\infty, \lim _{i \rightarrow \infty} x_{i}=x_{0}$ $\in G$.

Suppose that $y \in G$. We will prove that $\underline{H}_{f}(y)=\infty$. There is a domain $D$ such that $\bar{D}$ is compact in $G, y \in D$ and $x_{i}, x_{0} \in D$. By Lemma 2.11 we can restrict to a sublcass $\mathscr{L}_{\bar{D}} \subset \mathscr{L}_{f}$, which is uniformly bounded from below in $\bar{D}$. For that reason we may assume that the functions in $\mathscr{L}_{\bar{D}}$ are non-negative in $D$. Choose balls $\bar{B}^{n}\left(z_{j}, 2 r_{j}\right) \subset D$, $j=1, \ldots, k$, with the following properties.

(i) $x_{i}, x_{0} \in B^{n}\left(z_{1}, r_{1}\right)$, for $i>i_{0}$,

(ii) $y \in B^{n}\left(z_{k}, r_{k}\right)$,

(iii) $B^{n}\left(z_{j}, r_{j}\right) \cap B^{n}\left(z_{j+1}, r_{j+1}\right) \neq \varnothing, j=1, \ldots, r-1$.

Let $i>i_{0}$ and define the functions $\mathscr{U}_{i}^{j}$ as follows: $\mathscr{U}_{i}^{1}=u_{i}, \mathscr{U}_{i}^{j+1}=$ $P\left(\mathscr{U}_{i}^{j}, B^{n}\left(z_{j}, 2 r_{j}\right)\right), j=1, \ldots, k-1$. By iterated use of Harnacks's inequality for the functions $\mathscr{U}_{i}^{j}, j=1, \ldots, k$, it is easy to see that there is a constant $c>0$ independent of $i$ such that

$$
u_{i}\left(x_{i}\right) \leq \frac{1}{c} \mathscr{U}_{i}^{k}(y) \leq \frac{1}{c} \underline{H}_{f}(y) .
$$

By letting $i \rightarrow \infty$ we obtain $\underline{H}_{f}(y)=\infty$.

Next assume that $\underline{H}_{f}$ is locally bounded from above. According to Lemma 2.12 either $\underline{H}_{f}$ is continuous or identically $-\infty$. In the latter case the proof is complete. Suppose that $\underline{H}_{f}$ is continuous. Let $\bar{B}^{n}\left(x_{0}, r\right) \subset G$ and choose $C=\bar{B}^{n}\left(x_{0}, r\right)$ in Lemma 2.14. Lemma 2.14 shows that there is a sequence $v_{i} \in \mathscr{L}_{\bar{D}}$ such that $v_{i}>\underline{H}_{f}-1 / i$ in $\bar{B}^{n}\left(x_{0}, r\right)$. Consider the functions $V_{i}=P\left(v_{i}, B^{n}\left(x_{0}, r\right)\right)$. Again we have $\lim _{i \rightarrow \infty} V_{i}=\underline{H}_{f}$ uniformly in $\bar{B}^{n}\left(x_{0}, r\right)$. It follows from Harnack's principle [GLM1, Theorem 4.21], that $\underline{H}_{f}$ is an $F$-extremal in $B^{n}\left(x_{0}, r\right)$ and thus in $G$.

3. Regular boundary points. As in the classical harmonic case it is possible to define a barrier function for the boundary value problem of $F$-extremals. In this chapter we show that it gives a necessary and 
sufficient condition for the regularity of boundary points. The proof for necessity differs considerably from the linear situation. Our variational principle also gives a new proof for Bouligand's theorem [H, p. 169].

Let $G \subset R^{n}$ be a bounded domain. A point $x_{0} \in \partial G$ has an $F$-barrier if there exists a sub- $F$-extremal $w: G \rightarrow R$ such that

(a) $\overline{\lim }_{x \rightarrow y} w(x)<0$ for all $y \in \partial G, y \neq x_{0}$,

(b) $\lim _{x \rightarrow x_{0}} w(x)=0$.

3.1. THEOREM. Suppose that $f: \partial G \rightarrow R$ is bounded and continuous at $x_{0} \in \partial G$. If $x_{0}$ has an F-barrier, then

$$
\lim _{x \rightarrow x_{0}} \underline{H}_{f}(x)=f\left(x_{0}\right) .
$$

Proof. The proof is completely analogous to the classical proof. Let $\varepsilon>0$ and $M=\sup |f|$. By virtue of the assumptions there are constants $\delta>0$ and $k<0$ such that $\left|f(x)-f\left(x_{0}\right)\right|<\varepsilon$ if $\left|x-x_{0}\right|<\delta$ and $k w(x)$ $\geq 2 M$ if $\left|x-x_{0}\right| \geq \delta$. Note that the functions $f\left(x_{0}\right)+\varepsilon+k w$ and $f\left(x_{0}\right)-\varepsilon-k w$ belong to the classes $\mathscr{U}_{f}$ and $\mathscr{L}_{f}$ respectively. Observe that $k w$ is a super- $F$-extremal and $-k w$ is a sub- $F$-extremal. Then

$$
f\left(x_{0}\right)-\varepsilon-k w(x) \leq \underline{H}_{f}(x) \leq \bar{H}_{f}(x) \leq f\left(x_{0}\right)+\varepsilon+k w(x)
$$

or

$$
\left|\underline{H}_{f}(x)-f\left(x_{0}\right)\right| \leq \varepsilon+k w(x) .
$$

Since $w(x) \rightarrow 0$ as $x \rightarrow x_{0}$ we obtain $\underline{H}_{f}(x) \rightarrow f\left(x_{0}\right)$ as $x \rightarrow x_{0}$.

3.2. Definition. A bounded domain $G \subset R^{n}$ is called $F$-regular, if for all continuous $f: \partial G \rightarrow R$ there is an $F$-extremal $u \in C(\bar{G})$ with $u \mid \partial G=f$.

3.3. LEMMA. A domain $G \subset R^{n}$ is F-regular if and only if $\lim _{x \rightarrow y} \underline{H}_{f}(x)=f(y)$ for all $y \in \partial G$.

Proof. Suppose that $G$ is $F$-regular. Then for $f \in C(\partial G)$ there is $u$ as in Definition 3.2. Since $u \in \mathscr{L}_{f}$ it follows that $\underline{H}_{f} \geq u$ in $G$. Let $v \in \mathscr{L}_{f}$ and $y \in \partial G$. Now

$$
\varlimsup_{x \rightarrow y} v(x) \leq f(y)=\lim _{x \rightarrow y} u(x)
$$

and the $F$-comparison principle implies $v \leq u$ in $G$. Then $\underline{H}_{f}=$ $\sup \left\{v \mid v \in \mathscr{L}_{f}\right\} \leq u$ in $G$. We have proved that $\underline{H}_{f}=u$ and thus $\lim _{x \rightarrow y} \underline{H}_{f}(x)=\lim _{x \rightarrow y} u(x)=f(y)$ for all $y \in \partial G$. The converse is trivial. 
3.4. Definition. A boundary point $x_{0}$ of a bounded domain $G \subset R^{n}$ is called $F$-regular, if for all continuous $f: \partial G \rightarrow R$

$$
\lim _{x \rightarrow x_{0}} \underline{H}_{f}(x)=f\left(x_{0}\right)
$$

Lemma 3.3 implies

3.5. Corollary. A bounded domain $G$ is F-regular if and only if each boundary point of $G$ is $F$-regular.

Theorem 3.1 gives

3.6. Corollary. A point $x_{0} \in \partial G$ is F-regular if it has an $F$-barrier.

The converse of Corollary 3.6 is also true.

3.7. TheOREM. A point $x_{0} \in \partial G$ is F-regular if and only if $x_{0}$ has an F-barrier.

Proof. Suppose that $x_{0} \in \partial G$ has an $F$-barrier. It follows from Corollary 3.6 that $x_{0}$ is $F$-regular. To show the converse assume that $x_{0} \in \partial G$ is $F$-regular. Let $\bar{G} \subset B^{n}\left(x_{0}, R\right)$. We shall construct a barrier function $w$ at $x_{0}$. For this purpose we need a continuous sub- $F$-extremal $u$ in $B^{n}\left(x_{0}, R\right)$ such that $u\left(x_{0}\right)=0$ and $u(x)>0$ for $x \in B^{n}\left(x_{0}, R\right)$. The function $u$ is constructed as a solution of an obstacle problem.

We will use the function $\varphi=\left|x-x_{0}\right|$ as an obstacle. Let $B=$ $B^{n}\left(x_{0}, R\right)$ and

$$
\mathscr{F}(\varphi)=\left\{v \in C(\bar{B}) \cap W_{n}^{1}(B) \mid v \leq \varphi \text { in } B, v=\varphi \text { in } \partial B\right\} .
$$

There exists $u \in \mathscr{F}(\varphi)$ such that $I_{F}(u, B)=\inf \left\{I_{F}(v, B) \mid v \in \mathscr{F}(\varphi)\right\}$, see [GLM1, Theorem 5.15]. Now [GLM1, Theorem 5.17(ii)] implies that the function $u$ is a sub- $F$-extremal.

In what follows we will show that $u\left(x_{0}\right)=0$ and $u(x)>0$ for $x \in \bar{B}$, $x \neq x_{0}$. The function $h=\max \{u, 0\}$ belongs to the class $\mathscr{F}(\varphi)$, hence $u\left(x_{0}\right)=0$ and $u \geq 0$ in $B^{n}\left(x_{0}, R\right)$. Suppose that there is $x_{1} \in B^{n}\left(x_{0}, R\right)$ such that $u\left(x_{1}\right)=0$ and $x_{1} \neq x_{0}$. Now $x_{1} \in \tilde{A}$, where $\tilde{A}$ is a component of the open set $\left\{x \in B^{n}\left(x_{0}, R\right) \mid u(x)<\varphi(x)\right\}$. Observe that $u$ is an $F$-extremal in the set $\tilde{A}$ [GLM1, pp. 39-40]. Harnack's inequality implies that $u(x)=0$ for $x \in \tilde{A}$. This is a contradiction. Hence $u(x)>0$ for $x \in B^{n}\left(x_{0}, R\right) \backslash\left\{x_{0}\right\}$. 
We are ready to construct the barrier at $x_{0} \in \partial G$. Consider the function $\underline{H}_{u}$. Now $u \in \mathscr{L}_{u}$ and hence $\underline{H}_{u} \geq u$ in $G$. This yields

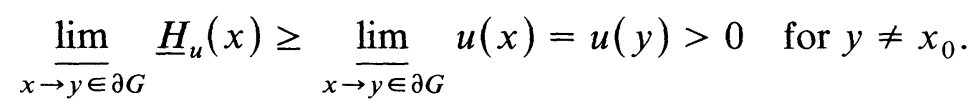

Since $x_{0}$ is $F$-regular it follows from Definition 3.4 that $\lim _{x \rightarrow x_{0}} \underline{H}_{u}(x)=$ $u\left(x_{0}\right)=0$.

For the barrier we choose the function $-\underline{H}_{u}$.

3.8. REMARK. The function $\underline{H}_{u}$ is the barrier sought in Bouligand's theorem.

4. Approximation of sub- $F$-extremals. In the classical potential theory it is well-known that subharmonic functions can be approximated by regular subharmonic functions. The following theorem gives a corresponding approximation result for sub- $F$-extremals. In particular, it follows that a general sub- $F$-extremal which is locally bounded from below is in the Sobolev-space loc $W_{n}^{1}(G)$.

4.1. Theorem. Suppose $u: G \rightarrow R \cup\{-\infty\}$ is a sub-F-extremal and $D \subset \subset G$ a domain. Then there exists a decreasing sequence of sub-F-extremals $u_{i} \in C(\bar{D}) \cap W_{n}^{1}(D)$ such that $\lim _{i \rightarrow \infty} u_{i}=u$ in $D$. If $u$ is locally bounded from below then $u$ is in $\operatorname{loc} W_{n}^{1}(G)$ and

$$
\int_{\text {spt } \eta} F(x, \nabla u) d m \leq \int_{\text {spt } \eta} F(x, \nabla(u-\eta)) d m,
$$

for all non-negative $\eta \in C_{0}^{\infty}(G)$.

Proof. Since $u$ is upper semicontinuous there exists a decreasing sequence $\varphi_{i} \in C^{\infty}(D) \cap C(\bar{D})$ such that $\lim _{i \rightarrow \infty} \varphi_{i}=u$ in $\bar{D}$. We may assume that the domain $D$ is regular. We shall again employ the solutions of an obstacle problem. Choose functions $u_{i}$ which minimize the integral

$$
I_{F}(u, D)=\int_{D} F(x, \nabla u) d m
$$

in the class $\mathscr{F}\left(\varphi_{i}\right)=\left\{u \in C(\bar{D}) \cap W_{n}^{1}(D) \mid u \leq \varphi_{i}\right.$ in $D, u=\varphi_{i}$ in $\left.\partial D\right\}$, see [GLM1, Theorem 5.15]. The functions $u_{i}$ are sub- $F$-extremals.

Next we show that $u \leq u_{i} \leq \varphi_{i}$ in $\bar{D}$. Consider the set $A_{i}=\{x \in$ $\left.D \mid u_{i}(x)<\varphi_{i}(x)\right\}$. Let $\tilde{A}_{i}$ be a component of $A_{i}$. Then $u_{i}$ is an $F$-extremal in $\tilde{A}_{i}$, see [GLM1, the proof of Theorem 5.17], and $u_{i}\left|\partial \tilde{A}_{i}=\varphi_{i}\right| \partial \tilde{A}_{i}$ $\geq u \mid \partial \tilde{A}_{i}$. By the $F$-comparison principle $u_{i} \geq u$ in $\tilde{A}_{i}$ and clearly in the whole $\bar{D}$. Thus $u=\lim _{i \rightarrow \infty} \varphi_{i} \geq \lim _{i \rightarrow \infty} u_{i} \geq u$ in $\bar{D}$. 
Next we prove that the sequence $u_{i}$ is decreasing. Assume the contrary. Then the open set $A=\left\{x \in D \mid u_{i+1}(x)>u_{i}(x)\right\}$ is non-empty for some $i$. The function $\min \left(u_{i}, u_{i+1}\right)$ belongs to the class $\mathscr{F}\left(\varphi_{i+1}\right)$. Now

$$
\begin{aligned}
I_{F}\left(u_{i+1}, D\right) & =I_{F}\left(u_{i+1}, A\right)+I_{F}\left(u_{i+1}, D \backslash A\right) \\
& \leq I_{F}\left(u_{i}, A\right)+I_{F}\left(u_{i+1}, D \backslash A\right)
\end{aligned}
$$

and hence $I_{F}\left(u_{i+1}, A\right) \leq I_{F}\left(u_{i}, A\right)$. In the same way we obtain

$$
\begin{aligned}
I_{F}\left(u_{i}, D\right) & =I_{F}\left(u_{i}, A\right)+I_{F}\left(u_{i}, D \backslash A\right) \\
& \leq I_{F}\left(\max \left(u_{i}, u_{i+1}\right), D\right) \\
& =I_{F}\left(u_{i+1}, A\right)+I\left(u_{i}, D \backslash A\right),
\end{aligned}
$$

i.e. $I_{F}\left(u_{i}, A\right) \leq I_{F}\left(u_{i+1}, A\right)$. Thus $I_{F}\left(u_{i}, A\right)=I_{F}\left(u_{i+1}, A\right)$ and it follows from the strict convexity of the kernel $F$ that the set $A$ is empty and $u_{i+1} \leq u_{i}$ in $D$.

Suppose $u$ is locally bounded from below. We prove that $u$ is in loc $W_{n}^{1}(D)$. Since $u_{i} \in C(\bar{D}) \cap W_{n}^{1}(D)$, [GLM1, Theorem 5.17] implies that

$$
\int_{\mathrm{spt} \eta} F\left(x, \nabla u_{i}\right) d m \leq \int_{\text {spt } \eta} F\left(x, \nabla\left(u_{i}-\eta\right)\right) d m
$$

for all non-negative $\eta \in C_{0}^{\infty}(D)$. Since $u$ is locally bounded from below we may assume that it is non-negative in $D$. Then also the functions $u_{i}$ are non-negative. Let $\bar{B}^{n}\left(x_{0}, r\right) \subset D$ and consider the condenser $\left(D, \bar{B}^{n}\left(x_{0}, r\right)\right)$. Analogously to the proof of [GLM1, Lemma 4.2] it can be shown that

$$
\begin{aligned}
\int_{B^{n}\left(x_{0}, r\right)}\left|\nabla u_{i}\right|^{n} d m & \leq \operatorname{cosc}\left(u_{i}, D\right)^{n} \operatorname{cap}_{n}\left(D, B^{n}\left(x_{0}, r\right)\right) \\
& \leq L \operatorname{cap}_{n}\left(D, B^{n}\left(x_{0}, r\right)\right)
\end{aligned}
$$

where the constant $L$ does not depend on $i$. This shows that the $L^{n}$-norms of $\nabla u_{i}$ are uniformly bounded. Hence there is a subsequence of $\nabla u_{i}$ converging weakly in $L^{n}\left(B^{n}\left(x_{0}, r\right)\right)$ to the generalized gradient $\nabla u$ of $u$, which is in $L^{n}\left(B^{n}\left(x_{0}, r\right)\right)$. Since the ball $B^{n}\left(x_{0}, r\right)$ was arbitrary, $u$ belongs to loc $W_{n}^{1}(D)$.

In order to prove the inequality (4.2) we show that there is a subsequence of $\nabla u_{i}$ such that $\nabla u_{i} \rightarrow \nabla u$ a.e. in compact subsets of $D$. The expression

$$
\left(\nabla_{h} F\left(x, h_{1}\right)-\nabla_{h} F\left(x, h_{2}\right)\right) \cdot\left(h_{1}-h_{2}\right),
$$


is strictly positive for a.e. $x \in G$, and all $h_{1}, h_{2} \in R^{n}, h_{1} \neq h_{2}$. Since the functions $u_{i}$ are sub- $F$-extremals in $D$ and belong to $C(\bar{D}) \cap W_{n}^{1}(D)$, they satisfy the inequality

$$
\int_{\text {spt } \eta} \nabla_{h} F\left(x, \nabla u_{i}\right) \cdot \nabla \eta d m \leq 0,
$$

for all non-negative $\eta \in C_{0}^{\infty}(D)$.

Let $\bar{B}=\bar{B}^{n}\left(x_{0}, r\right) \subset D, 0<r^{\prime}<r, \zeta \in C_{0}^{\infty}(B), 0 \leq \zeta \leq 1$, and $\zeta(x)=1$ for $x \in B^{n}\left(x_{0}, r^{\prime}\right)$. Put $\eta=\zeta\left(u_{i}-u\right)$ and use (4.6) to obtain

$$
\begin{aligned}
\int_{\mathrm{spt} \zeta}\left(\nabla_{h} F(\right. & \left.\left.x, \nabla u_{i}\right)-\nabla_{h} F(x, \nabla u)\right) \cdot \nabla\left(\zeta\left(u_{i}-u\right)\right) d m \\
= & \int_{\mathrm{spt} \zeta} \zeta\left(\nabla_{h} F\left(x, \nabla u_{i}\right)-\nabla_{h} F(x, \nabla u)\right) \cdot\left(\nabla u_{i}-\nabla u\right) d m \\
& +\int_{\mathrm{spt} \zeta}\left(u_{i}-u\right)\left(\nabla_{h} F\left(x, \nabla u_{i}\right)-\nabla_{h} F(x, \nabla u)\right) \cdot \nabla \zeta d m \\
= & I_{i}^{1}+I_{i}^{2} \leq-\int_{\mathrm{spt} \zeta} \nabla_{h} F(x, \nabla u) \cdot \nabla\left(\zeta\left(u_{i}-u\right)\right) d m \\
= & -\int_{\mathrm{spt} \zeta}\left(u_{i}-u\right) \nabla_{h} F(x, \nabla u) \cdot \nabla \zeta d m \\
& -\int_{\mathrm{spt} \zeta} \zeta \nabla_{h} F(x, \nabla u) \cdot\left(\nabla u_{i}-\nabla u\right) d m .
\end{aligned}
$$

Because of the inequality (4.4) we can choose a subsequence of $u_{i}$ such that $u_{i} \rightarrow u$ in $L^{n}\left(B^{n}\left(x_{0}, r\right)\right)$ and $\nabla u_{i} \rightarrow \nabla u$ weakly in $L^{n}\left(B^{n}\left(x_{0}, r\right)\right)$, see [M, p. 75, Theorem 3.4.4]. Then the last two integrals and the integral $I_{i}^{2}$ tend to zero for $i \rightarrow \infty$. Now (4.5) yields $I_{i}^{1} \geq 0$ and hence $\lim _{i \rightarrow \infty} I_{i}^{1}=0$. Then we employ the condition (4.5) to show that there is a subsequence of $\nabla u_{i}$ such that $\nabla u_{i} \rightarrow \nabla u$ for a.e. $x \in B^{n}\left(x_{0}, r^{\prime}\right)$. Write

$$
g_{i}(x)=\left(\nabla_{h} F\left(x, \nabla u_{i}(x)\right)-\nabla_{h} F(x, \nabla u(x))\right) \cdot\left(\nabla u_{i}(x)-\nabla u(x)\right) \text {. }
$$

Then $g_{i} \rightarrow 0$ in $L^{1}\left(B^{n}\left(x_{0}, r^{\prime}\right)\right)$ and hence there is a subsequence such that $g_{i}(x) \rightarrow 0$ for a.e. $x \in B^{n}\left(x_{0}, r^{\prime}\right)$. It follows from (4.5) that $\nabla u_{i}(x) \rightarrow$ $\nabla u(x)$ for a.e. $x \in B^{n}\left(x_{0}, r^{\prime}\right)$.

Finally choose a non-negative $\eta \in C_{0}^{\infty}\left(B^{n}\left(x_{0}, r^{\prime}\right)\right)$ in (4.6). Since the integrals

$$
\int_{B^{n}\left(x_{0}, r^{\prime}\right)}\left|\nabla_{h} F\left(x, \nabla u_{i}\right)\right|^{n /(n-1)} d m
$$


are uniformly bounded and $\nabla_{h} F\left(x, \nabla u_{i}(x)\right) \rightarrow \nabla_{h} F(x, \nabla u(x))$ a.e. in $B^{n}\left(x_{0}, r^{\prime}\right)$, the inequality (4.6) yields via weak convergence

$$
\int_{B^{n}\left(x_{0}, r^{\prime}\right)} \nabla_{h} F(x, \nabla u) \cdot \nabla \eta d m \leq 0 .
$$

Thus the above inequality holds in $D$ and (4.2) follows from [GLM1, Theorem 5.17].

5. F-harmonic measure. The PWB-method can be used in the definition of the $F$-harmonic measure. In [GLM2] the $F$-harmonic measure was constructed via generating sequences. This method can only be used in regular domains.

Suppose $G \subset R^{n}$ is a bounded open set. Let $C \subset \partial G$ be a closed set and let $f: \partial G \rightarrow R$ be the characteristic function of $C$. The function $\bar{H}_{f}$, which is an $F$-extremal, is the $F$-harmonic measure of $C$ with respect to $G$. The next theorem shows that in regular domains this concept gives the same $F$-harmonic measure.

5.1. Theorem. Suppose that $G \subset R^{n}$ is a regular domain, and that $C \subset \partial G$ is a closed set. If $f$ is the characteristic function of $C$, then $\bar{H}_{f}=\omega(C, G ; F)$, where $\omega(C, G ; F)$ is the F-harmonic measure as in [GLM2, Definition 2.16].

Proof. Let $\varphi_{i}$ be a $(C, G)$-boundary sequence, see [GLM2, pp. 235-236]. Consider the $F$-extremals $u_{i} \in C(\bar{G}) \cap W_{n}^{1}(G)$ with $u_{i} \mid \partial G=$ $\varphi_{i} \mid \partial G$. It was shown in [GLM2, pp. 3-4] that $\lim _{i \rightarrow \infty} u_{i}=\omega(C, G ; F)$ locally uniformly in $G$. Now $u_{i} \in \mathscr{U}_{f}$ and hence $u_{i} \geq \bar{H}_{f}$. Thus $\omega(C, G ; F)=\lim _{i \rightarrow \infty} u_{i} \geq \bar{H}_{f}$. On the other hand, for $u \in \mathscr{U}_{f}$, $\lim _{x \rightarrow y \in \partial G} u(x) \geq f(y) \geq \varlimsup_{x \rightarrow y \in \partial G} \omega(C, G ; F)$, see [GLM2, Remark 2.20]. Lemma 2.3 implies that $u \geq \omega(C, G ; F)$, in $G$ and thus $\bar{H}_{f} \geq$ $\omega(C, G ; F)$, which together with the previous inequality completes the proof.

\section{REFERENCES}

[BJ] E. F. Beckenbach, and L. K. Jackson, Subfunctions of several variables, Pacific J. Math., 3 (1953), 291-313.

[CC] C. Constantinescu and A. Cornea, Potential Theory on Harmonic Spaces, Springer-Verlag, 1972.

[GZ] R. Gariepy, and W. P. Ziemer, A regularity condition at the boundary for solutions of quasilinear elliptic equations, Arch. Rational Mech. Anal., 67 (1977), 25-39.

[GLM1] S. Granlund, P. Lindqvist and O. Martio, Conformally invariant variational integrals, Trans. Amer. Math. Soc., 277 (1983), 43-73. 
[GLM2] __ F-harmonic measure in space, Ann. Acad. Sci. Fenn. A I Math., 7 (1982), 233-247.

[GLM3] _ Phragmén-Lindelöf's and Lindelöf's theorems, Arkiv für Mat., 231 (1985), 103-128.

[H] L. L. Helms, Introduction to Potential Theory, Wiley, 1969.

[J] L. K. Jackson, On generalized subharmonic functions, Pacific J. Math., 5 (1955), 215-228.

[M] C. B. Morrey, Multiple Integrals in the Calculus of Variations, Springer-Verlag, 1966.

[P] O. Perron, Eine neue Behandlung der ersten Randwertaufgabe für $\Delta u=0$, Math. Z., 18 (1923), 42-54.

[R] T. Rado, Subharmonic Functions, Ergebnisse der Mathematik und Ihrer Grenzgebiete, Chelsea Publishing Company, 1949.

Received May 27, 1983 and in final form January 29, 1986.

HeLSINKI UNIVERSITY OF TECHNOLOGY

INSTITUTE OF MATHEMATICS

SF-02150 ESPOO 15

FINLAND

AND

UNIVERSITY OF JYVÄSKYLÄ

DEPARTMENT OF MATHEMATICS

SF-40100 JYVÄSKYLÄ 10

FINLAND 



\title{
PACIFIC JOURNAL OF MATHEMATICS \\ EDITORS
}

\author{
V. S. VARADARAJAN \\ (Managing Editor) \\ University of California \\ Los Angeles, CA 90024 \\ HERBERT CLEMENS \\ University of Utah \\ Salt Lake City, UT 84112 \\ R. FINN \\ Stanford University \\ Stanford, CA 94305
}

\author{
HERMANN FLASCHKA \\ University of Arizona \\ Tucson, AZ 85721
}

RAMESh A. GANGOLLI

University of Washington Seattle, WA 98195

VAUGHAN F. R. JONES

University of California

Berkeley, CA 94720

ROBION KIRBY

University of California

Berkeley, CA 94720
C. C. MOORE

University of California

Berkeley, CA 94720

H. SAMELSON

Stanford University

Stanford, CA 94305

HAROLD STARK

University of California, San Diego

La Jolla, CA 92093

\section{ASSOCIATE EDITORS}

\author{
R. ARENS \\ E. F. BECKENBACH \\ B. H. NeUmanN \\ F. WOLF \\ K. YOSHIDA \\ (1906-1982)

\section{SUPPORTING INSTITUTIONS} \\ UNIVERSITY OF ARIZONA \\ UNIVERSITY OF BRITISH COLUMBIA \\ CALIFORNIA INSTITUTE OF TECHNOLOGY \\ UNIVERSITY OF CALIFORNIA \\ MONTANA STATE UNIVERSITY \\ UNIVERSITY OF NEVADA, RENO \\ NEW MEXICO STATE UNIVERSITY \\ OREGON STATE UNIVERSITY \\ UNIVERSITY OF OREGON \\ UNIVERSITY OF SOUTHERN CALIFORNIA \\ STANFORD UNIVERSITY \\ UNIVERSITY OF HAWAII \\ UNIVERSITY OF TOKYO \\ UNIVERSITY OF UTAH \\ WASHINGTON STATE UNIVERSITY \\ UNIVERSITY OF WASHINGTON
}

The Supporting Institutions listed above contribute to the cost of publication of this Journal, but they are not owners or publishers and have no responsibility for its content or policies.

Mathematical papers intended for publication in the Pacific Journal of Mathematics should be in typed form or offset-reproduced (not dittoed), double spaced with large margins. Please do not use built up fractions in the text of the manuscript. However, you may use them in the displayed equations. Underline Greek letters in red, German in green, and script in blue. The first paragraph must be capable of being used separately as a synopsis of the entire paper. In particular it should contain no bibliographic references. Please propose a heading for the odd numbered pages of less than 35 characters. Manuscripts, in triplicate, may be sent to any one of the editors. Please classify according to the scheme of Math. Reviews, Index to Vol. 39. Supply name and address of author to whom proofs should be sent. All other communications should be addressed to the managing editor, or Elaine Barth, University of California, Los Angeles, California 90024.

There are page-charges associated with articles appearing in the Pacific Journal of Mathematics. These charges are expected to be paid by the author's University, Government Agency or Company. If the author or authors do not have access to such Institutional support these charges are waived. Single authors will receive 50 free reprints; joint authors will receive a total of 100 free reprints. Additional copies may be obtained at cost in multiples of 50 .

The Pacific Journal of Mathematics is issued monthly as of January 1966. Regular subscription rate: $\$ 190.00$ a year (5 Vols., 10 issues). Special rate: $\$ 95.00$ a year to individual members of supporting institutions.

Subscriptions, orders for numbers issued in the last three calendar years, and changes of address should be sent to Pacific Journal of Mathematics, P.O. Box 969, Carmel Valley, CA 93924, U.S.A. Old back numbers obtainable from Kraus Periodicals Co., Route 100, Millwood, NY 10546.

The Pacific Journal of Mathematics at P.O. Box 969, Carmel Valley, CA 93924 (ISSN 0030-8730) publish'es 5 volumes per year. Application to mail at Second-class postage rates is pending at Carmel Valley, California, and additional mailing offices. Postmaster: send address changes to Pacific Journal of Mathematics, P.O. Box 969, Carmel Valley, CA 93924.

PUBLISHED BY PACIFIC JOURNAL OF MATHEMATICS, A NON-PROFIT CORPORATION

Copyright (C) 1986 by Pacific Journal of Mathematics 


\section{Pacific Journal of Mathematics}

\section{Vol. 125, No. $2 \quad$ October, 1986}

Dale Edward Alspach, On $\mathscr{L}_{p, \lambda}$ spaces for small $\lambda \ldots \ldots \ldots \ldots \ldots \ldots 257$

Jong Sook Bae and Sangsuk Yie, Range of Gateaux differentiable operators

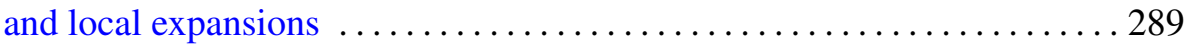

Hubert Berens and Lothar Hetzelt, On accretive operators on $l_{n}^{\infty} \ldots \ldots 301$

Marie-Françoise Bidaut-Véron, Global existence and uniqueness results for singular solutions of the capillarity equation ................. 317

Donald M. Davis and Mark Mahowald, Classification of the stable homotopy types of stunted real projective spaces ................. 335

Aad Dijksma, Heinz K. Langer and Hendrik S. V. de Snoo, Unitary

colligations in $\Pi_{\kappa}$-spaces, characteristic functions and Štraus extensions

Michel Enock and Jean-Marie Schwartz, Algèbres de Kac

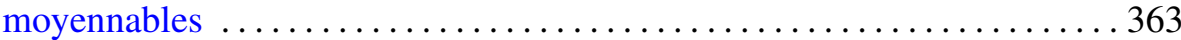

Seppo Granlund, Peter Lindqvist and Olli Martio, Note on the

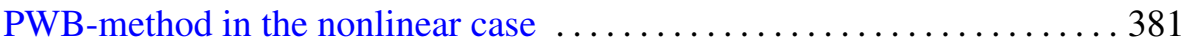

Palle E. T. Jorgensen, Analytic continuation of local representations of Lie

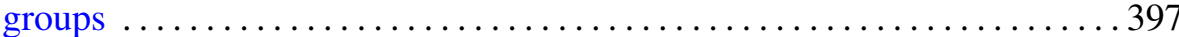

Robert P. Kaufman, Plane curves and removable sets ................409

José M. Montesinos and Wilbur Carrington Whitten, Constructions of two-fold branched covering spaces $\ldots \ldots \ldots \ldots \ldots$.

Benedict Seifert, Highly transitive group actions on trees and normalizing Tits systems

Charles Stuart Stanton, Counting functions and majorization for Jensen

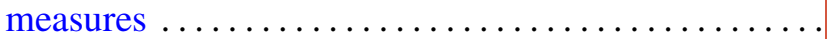

Luen-Fai Tam, On existence criteria for capillary free surfaces without gravity

Zhuocheng Yang, Exposed points of left invariant means 